\title{
On Bargaining Sets of Convex NTU Games
}

\author{
by \\ Bezalel Peleg \\ and \\ Peter Sudhölter
}

Discussion Papers on Business and Economics

No. 19/2014

FURTHER INFORMATION Department of Business and Economics Faculty of Business and Social Sciences University of Southern Denmark Campusvej 55 DK-5230 Odense M Denmark

Tel.: +456550 3271

Fax: +456550 3237

E-mail: 1ho@sam.sdu.dk http://www.sdu.dk/ivoe 


\title{
On Bargaining Sets of Convex NTU Games*
}

\author{
Bezalel Peleg ${ }^{\dagger} \quad$ Peter Sudhölter ${ }^{\ddagger}$
}

\begin{abstract}
We show that the Aumann-Davis-Maschler bargaining set and the Mas-Colell bargaining set of a non-leveled NTU game that is either ordinal convex or coalition merge convex coincides with the core of the game. Moreover, we show by means of an example that the foregoing statement may not be valid if the NTU game is marginal convex.
\end{abstract}

Keywords: NTU game $\cdot$ Convex game $\cdot$ Bargaining set

\section{JEL Classification: C71}

\section{Introduction}

Convex TU games were introduced by Shapley (1971) who discussed their basic properties and applications. One distinguished property of the family of convex games is that many of the leading solutions of TU games coincide on it. For example, Shapley has already proved in his aforementioned paper that the (non empty) core of a convex game coincides with its (unique) von Neumann Morgenstern solution. Clearly, this result makes the core look more intuitive. Also Shapley showed, in the same paper, that the Shapley value of a convex game is a member of its core, which makes the value look more intuitive.

A second step was taken by Maschler, Peleg, and Shapley (1972) who proved that the kernel of a convex TU game coincides with its nucleolus and the core coincides with its (AumannDavis-Maschler) bargaining set. Clearly these results enforce the intuitive meaning of both the core and the bargaining set. (Indeed, Maschler (1976) claims that for some games the AumannDavis-Maschler bargaining set has an advantage over the core.) This paper is the starting point of our investigation: We inquire whether the core and various bargaining sets coincide for convex NTU games.

\footnotetext{
${ }^{*}$ Peter Sudhölter was supported by The Danish Council for Independent Research|Social Sciences under the FINQ project and by the Spanish Ministerio de Ciencia e Innovación under project ECO2012-33618.

${ }^{\dagger}$ Institute of Mathematics and The Federmann Center for the Study of Rationality, The Hebrew University of Jerusalem, Feldman Building, Givat Ram, 91904 Jerusalem, Israel. E-mail: pelegba@math.huji.ac.il

${ }^{\ddagger}$ Department of Economics and COHERE, University of Southern Denmark, Campusvej 55, 5230 Odense M, Denmark. E-mail: psu@sam.sdu.dk
} 
Ordinal convexity for NTU games was introduced by Vilkov (1977) who generalized some of Shapley's (1971) results (under restrictive conditions). Peleg (1986) proved that the core of an ordinal convex NTU game coincides with the von Neumann Morgenstern solution. In this paper we investigate the bargaining set and the Mas-Colell bargaining set of ordinal convex and coalition merge convex NTU games (see Sections 2 and 4 for the terminology), and prove their coincidence with the core (under the assumption of non-levelness).

\section{Preliminaries}

Let $N$ be a finite nonempty set. For $S \subseteq N$ we denote by $\mathbb{R}^{S}$ the set of all real functions on $S$. If $x, y \in \mathbb{R}^{S}$, then we write $x \geqslant y$ if $x_{i} \geqslant y_{i}$ for all $i \in S$. Moreover, we write $x>y$ if $x \geqslant y$ and $x \neq y$ and we write $x \gg y$ if $x^{i}>y^{i}$ for all $i \in S$. Denote $\mathbb{R}_{+}^{S}=\left\{x \in \mathbb{R}^{S} \mid x \geqslant 0\right\}$. A set $C \subseteq \mathbb{R}^{S}$ is comprehensive if $x \in C, y \in \mathbb{R}^{S}$, and $y \leqslant x$ imply that $y \in C$. An NTU game with the player set $N$ is a pair $(N, V)$ where $V$ is a function which associates with every coalition $S$ (that is, $S \subseteq N$ and $S \neq \emptyset$ ) a set $V(S) \subseteq \mathbb{R}^{S}, V(S) \neq \emptyset$, such that

(1) $V(S)$ is closed and comprehensive;

(2) $V(S) \cap\left(x+\mathbb{R}_{+}^{S}\right)$ is bounded for every $x \in \mathbb{R}^{S}$.

Moreover, we assume that $V(\emptyset)=\emptyset$.

Let $(N, V)$ be an NTU game. Abbreviating "boundary" by " $\partial$ " we have

$$
\partial V(N)=\{x \in V(N) \mid \text { there exists no } y \in V(N) \text { such that } y \gg x\}
$$

i.e., $\partial V(N)$ is the set of weakly Pareto optimal elements of $V(N)$. Note that for any $\emptyset \neq S \subseteq N$, $x \in \mathbb{R}^{S}$ is Pareto optimal in $V(S)$ if $x \in V(S)$ and if $y \in V(S)$ and $y \geqslant x$ imply $x=y$. Note that, if $(N, V)$ is non-leveled, i.e., for all $\emptyset \neq S \subseteq N$ and all $x, y \in \partial V(S), x \geqslant y$ implies $x=y$, then $\partial V(S)$ is the set of Pareto optimal elements in $V(S)$.

In order to recall the definitions of the unconstrained (Aumann-Davis-Maschler) bargaining set (Aumann and Maschler 1964, Davis and Maschler 1967) and of the Mas-Colell prebargaining set (Mas-Colell 1989), let $x \in \mathbb{R}^{N}$. A pair $(P, y)$ is an objection at $x$ if $\emptyset \neq P \subseteq N, y$ is Pareto optimal in $V(P)$, and $y>x_{P}$. An objection $(P, y)$ is strong if $y \gg x_{P}$. The pair $(Q, z)$ is a weak counter objection to the objection $(P, y)$ if $Q \subseteq N, Q \neq \emptyset, P$, if $z \in V(Q)$, and if $z \geqslant\left(y_{P \cap Q}, x_{Q \backslash P}\right)$. A weak counter objection $(Q, z)$ is a counter objection to the objection $(P, y)$ if $z>\left(y_{P \cap Q}, x_{Q \backslash P}\right)$. A strong objection $(P, y)$ is justified in the sense of the bargaining set if there exist players $k \in P$ and $\ell \in N \backslash P$ such that there does not exist any weak counter objection $(Q, z)$ to $(P, y)$ satisfying $\ell \in Q$ and $k \notin Q$. The unconstrained bargaining set of $(N, V), \mathcal{P} \mathcal{M}(N, V)$, is the set of all $x \in \partial V(N)$ that do not have strong justified objections at $x$ in the sense of the bargaining set (Davis and Maschler 1967). An objection (P,y) is justified in the sense of the Mas-Colell bargaining set if there does not exist any counter objection to $(P, y)$. The Mas-Colell prebargaining set of $(N, V), \mathcal{P} \mathcal{M B}(N, V)$, is the set of all $x \in \partial V(N)$ that do 
not have a justified objection at $x$ in the sense of the Mas-Colell bargaining set (Mas-Colell 1989).

Note that the bargaining set, $\mathcal{M}(N, V)$, is defined by $\mathcal{M}(N, V)=\mathcal{P} \mathcal{M}(N, V) \cap I(N, V)$ and the Mas-Colell bargaining set, $\mathcal{M B}(N, V)$, is defined by $\mathcal{M B}(N, V)=\mathcal{P} \mathcal{M B}(N, V) \cap I(N, V)$, where $I(N, V)=\left\{x \in \partial V \mid x_{i} \geqslant \max V(\{i\})\right.$ for all $\left.i \in N\right\}$, i.e., $I(N, V)$ is the set of imputations.

Recall that $(N, V)$ is

- superadditive if $V(S) \times V(T) \subseteq V(S \cup T)$ for all $S \subseteq N$ and $T \subseteq N \backslash S$;

- ordinal convex if for all $S, T \subseteq N$ and $x \in \mathbb{R}^{N}, x_{S} \in V(S)$ and $x_{T} \in V(T)$ imply that $x_{S \cap T} \in V(S \cap T)$ or $x_{S \cup T} \in V(S \cup T)$.

Note that an ordinal convex game is, hence, superadditive.

\section{The excess NTU game}

For an NTU game $(N, V)$ and $x \in \mathbb{R}^{N}$ we define the excess game $\left(N, V^{x}\right)$ by the requirement that, for any $\emptyset \neq S \subseteq N$,

$$
V^{x}(S)=\left(-\mathbb{R}_{+}^{S}\right) \cup \bigcup_{\emptyset \neq T \subseteq S}\left(V(T)-x_{T}\right) \times\left(-\mathbb{R}_{+}^{S \backslash T}\right)
$$

Note that with $V^{x}(\emptyset)=\emptyset$ the pair $\left(N, V^{x}\right)$ is an NTU game (i.e., (1) and (2) are valid).

Lemma 3.1 Let $(N, V)$ be an NTU game and $x \in V(N)$. Then $x \in \mathcal{C}(N, V)$ if and only if $0 \in \mathcal{C}\left(N, V^{x}\right)$.

Proof: If $x \in \mathcal{C}(N, V)$, then $0=x-x \in V^{x}(N)$. Moreover, if $y \in V^{x}(S), y \notin 0 \in \mathbb{R}^{S}$, for some $\emptyset \neq S \subseteq N$, then there exists $\emptyset \neq R \subseteq S$ such that $y_{R}+x_{R} \in V(R)$ and $y_{S \backslash R} \leqslant 0$. Hence, there exists $i \in R$ such that $x_{i} \geqslant y_{i}+x_{i}$ and we conclude that $0 \in \mathcal{C}\left(N, V^{x}\right)$.

Conversely, if $0 \in \mathcal{C}\left(N, V^{x}\right)$, then, for any coalition $T$ and any $y \in V(T), y \geqslant x_{T}$ implies $y-x_{T} \in V^{x}(T)$ so that there exists $j \in T$ with $y_{j}-x_{j} \leqslant 0$. Thus, $x \in \mathcal{C}(N, V)$.

q.e.d.

We may now prove the main result of this section.

Theorem 3.2 If $(N, V)$ is a non-leveled game and $x \in \mathcal{P} \mathcal{M B}(N, V)$, then $\mathcal{C}\left(N, V^{x}\right) \neq \emptyset$ iff $x \in \mathcal{C}(N, V)$.

Proof: One direction is a direction of Lemma 3.1 (2). For the remaining direction, let $\bar{x} \in$ $\mathcal{C}\left(N, V^{x}\right)$ and assume, on the contrary, that $x \notin \mathcal{C}(N, V)$. Let $P=\left\{i \in N \mid \bar{x}_{i}>0\right\}$. As $V^{x}(\{i\}) \supseteq-\mathbb{R}_{+}$for all $i \in N, \bar{x} \geqslant 0$ and by the relevant direction of (2) of Lemma 3.1, $P \neq \emptyset$. As $\bar{x} \in V^{x}(N)$, there exists $P \subseteq S \subseteq N$ such that $\bar{x}_{S}+x_{S} \in V(S)$. Hence, $\left(P, \bar{x}_{P}+x_{P}\right)$ is an objection to $x$ in the sense of the Mas-Colell bargaining set. Let $(Q, y)$ be a counterobjection 
to $\left(P, \bar{x}_{P}+x_{P}\right)$ then $y>\left(\bar{x}_{P \cap Q}+x_{P \cap Q}, x_{Q \backslash P}\right)$. By non-levelness there exists $y^{\prime} \in V(Q)$ such that $y^{\prime} \gg\left(\bar{x}_{P \cap Q}+x_{P \cap Q}, x_{Q \backslash P}\right)$. As $\bar{x}_{Q \backslash P}=0, y^{\prime}-x_{Q} \in V^{x}(Q)$ and $y^{\prime}-x_{Q} \gg \bar{x}_{Q}$ which is impossible because $\bar{x} \in \mathcal{C}\left(N, V^{x}\right)$.

q.e.d.

The following corollary may be regarded as a generalization of Solymosi's (1999) main result for TU games.

Corollary 3.3 If $(N, V)$ is a superadditive non-leveled $N T U$ game and $x \in \mathcal{P} \mathcal{M}(N, V)$, then $\mathcal{C}\left(N, V^{x}\right) \neq \emptyset$ if and only if $x \in \mathcal{C}(N, V)$.

Proof: We may assume that $(N, V)$ is zero-normalized because the set of superadditive nonleveled NTU games on $N$ is closed under translations and the core and the unconstrained bargaining set are translation covariant. Then $(N, V)$ satisfies all assumptions of Holzman's (2001) Theorem 3.1 stating that $\mathcal{M}(N, V) \subseteq \mathcal{P} \mathcal{M B}(N, V)$. His proof, however, does not use individual rationality so that, in fact, $\mathcal{P} \mathcal{M}(N, V) \subseteq \mathcal{P} \mathcal{M B}(N, V)$, and Theorem 3.2 finishes the proof.

q.e.d.

\section{Results and examples}

In order to apply the results of Section 3 to ordinal convex NTU games, the following lemma is needed.

Lemma 4.1 If $(N, V)$ is an ordinal convex $N T U$ game, then $\left(N, V^{x}\right)$ is ordinal convex.

Proof: Let $\emptyset \neq S, T \subseteq N$ and let $y \in \mathbb{R}^{N}$ satisfy $y_{S} \in V^{x}(S)$ and $y_{T} \in V^{x}(T)$. We have to show that $y_{S \cap T} \in V^{x}(S \cap T)$ or $y_{S \cup T} \in V^{x}(S \cup T)$. If $y_{S} \leqslant 0 \in \mathbb{R}^{S}$ or $y_{T} \leqslant 0 \in \mathbb{R}^{T}$, then $y_{S \cup T} \in V^{x}(S \cup T)$. Hence, we may assume that neither $y_{S} \leqslant 0$ nor $y_{T} \leqslant 0$. Then there exist $Q \subseteq S$ and $R \subseteq T$ such that $Q \neq \emptyset \neq R, y_{Q} \in V(Q)-x_{Q}, y_{S \backslash Q} \leqslant 0, y_{R} \in V(R)-x_{R}$, and $y_{T \backslash R} \leqslant 0$. Therefore there exists $z \in \mathbb{R}^{N}$ such that $y \leqslant z-x, z_{Q} \in V(Q), z_{R} \in V(R), z_{i}=x_{i}$ for all $i \in(S \cup T) \backslash(Q \cup R)$. By ordinal convexity of $V, z_{Q \cap R} \in V(Q \cap R)$ or $z_{Q \cup R} \in V(Q \cup R)$. If $z_{Q \cap R} \in V(Q \cap R)$, then $z_{S \cap T}-x_{S \cap T} \in V^{x}(S \cap T)$ so that $y_{S \cap T} \in V^{x}(S \cap T)$ by comprehensiveness. Similarly, $z_{Q \cup R} \in V(Q \cup R)$ implies that $y_{S \cup T} \in V^{x}(S \cup T)$.

q.e.d.

The core of an ordinal convex game is nonempty (Greenberg 1985). Moreover, an ordinal convex game is superadditive. Thus, Theorem 3.2, Lemma 3.1, and Corollary 3.3 have the following consequence.

Corollary 4.2 The unconstrained bargaining set and the Mas-Colell prebargaining set of any ordinal convex non-leveled NTU game coincide with its core. 
The following example shows that "non-levelness" is needed in the statement concerning the bargaining set of Corollary 4.2. Let $|N| \geqslant 3$ and, for any $S \subseteq N$,

$$
V(S)= \begin{cases}\emptyset & , \text { if } S=\emptyset, \\ -\chi_{S}^{S}-\mathbb{R}_{+}^{S} & , \text { if } 1 \leqslant|S| \leqslant|N|-2, \\ -\mathbb{R}_{+}^{S} & , \text { if }|S| \geqslant|N|-1,\end{cases}
$$

where $\chi^{S} \in \mathbb{R}^{N}$ is the characteristic vector of $S$, i.e., $\chi_{i}^{S}=1$ for $i \in S$ and $\chi_{j}^{S}=0$ for $j \in N \backslash S$. Then $(N, V)$ is ordinal convex. Let $k \in N$ and $x=-\chi_{N \backslash\{k\}}$. Then $x \notin \mathcal{C}(N, V)$. Note that $k$ has no objection against any other player and any objection of any $i \in N \backslash\{k\}$ is of the form $(S, y)$ such that $S=N \backslash\{k\}, 0 \geqslant y \gg x_{N \backslash\{k\}}$, so that $(N \backslash\{i\}, 0)$ is a counterobjection. As $x$ is individually rational, $x \in \mathcal{M}(N, V)$.

By means of an example that is derived from the voting game of the Voting Paradox (Holzman, Peleg, and Sudhölter 2007, Sect. 3) we now show that nonlevelness is also crucial for the statement concerning the Mas-Colell bargaining set.

Example 4.3 Let $N=\{1,2,3\}$ and $(N, V)$ the 0-normalized game defined by

$$
\begin{aligned}
& V(\{1,2\})=\{(2,1),(0,2)\}-\mathbb{R}_{+}^{\{1,2\}}, \\
& V(\{1,3\})=\{(2,0),(1,2)\}-\mathbb{R}_{+}^{\{1,3\}}, \\
& V(\{2,3\})=\{(2,1),(0,2)\}-\mathbb{R}_{+}^{\{2,3\}}, \text { and } \\
& V(N) \quad=\{(2,2,0),(2,0,2),(0,2,2),(2,1,1),(1,2,1),(1,1,2)\}-\mathbb{R}_{+}^{N} .
\end{aligned}
$$

For any $\emptyset \neq S, T \subseteq N$ and any $x \in \mathbb{R}^{N}$ such that $x_{S} \in V(S)$ and $x_{T} \in V(T)$ we have $x_{S \cup T} \in V(S \cup T)$. Indeed, in order to verify this fact we may assume that $S \backslash T \neq \emptyset \neq T \backslash S$. If $|S|=|T|=1$, then $x_{S \cup T} \leqslant 0 \in V(S \cup T)$. Otherwise $S \cup T=N$. If $x \ngtr 0$, say $x_{i} \leqslant 0$, then $x_{j} \leqslant 2$ for all $j \in N$ implies $x \in V(N)$. Finally, if $x \gg 0$, then $|S|=|T|=2$ and $x \leqslant(2,1,1)$ or $x \leqslant(1,2,1)$ or $x \leqslant(1,1,2)$. Hence, $(N, V)$ is ordinal convex.

Let $y=(1,1,0)$. Then $y$ is weakly Pareto optimal. Assume that $y$ has a justified weak objection $(P, z)$. Then $z$ is Pareto optimal in $V(P)$. If $P=N$ and $z=(2,2,0), z=(2,0,2)$, or $z=(0,2,2)$, then $(P, z)$ can be countered by $(\{2,3\},(2,1)),(\{1,2\},(2,1))$, or $(\{1,3\},(1,2))$, respectively. If $z=(2,1,1), z=(1,1,2)$, or $z=(1,2,1)$, then $(P, z)$ can also be countered by the aforementioned pairs, respectively. If $P=\{1,2\}$, then $z=(2,1)$ so that $(\{2,3\},(2,1))$ is a counterobjection. If $P=\{2,3\}$, then $z=(2,1)$ so that $(\{1,3\},(1,2))$ is a counterobjection. Finally, if $P=\{1,3\}$, then either $z=(2,0)$ so that $(\{2,3\},(2,1))$ is a counterobjection or $z=(1,2)$ so that $(\{1,2\},(2,1))$ is a counterobjection. Hence $y \in \mathcal{M B}(N, V)$.

Moreover, $(2,1) \in V(\{2,3\})$. Thus $\mathcal{M B}(N, V) \backslash \mathcal{C}(N, V) \neq \emptyset$. Also, $(2,2,0) \in \mathcal{C}(N, V)$ has the justified objection $(\{2,3\},(2,1))$ so that $\mathcal{C}(N, V) \backslash \mathcal{M B}(N, V) \neq \emptyset$.

For $|N|=2$ the bargaining set $\mathcal{M}$ coincides with and the Mas-Colell prebargaining set $\mathcal{P} \mathcal{M B}$ is contained in the core, provided that the core is nonempty. If $(N, V)$ is defined by $V(S)=-\mathbb{R}^{S}$ for all $S \subseteq N$, then $\mathcal{C}(N, V)=\{0\}$ and 0 is the unique individually rational feasible payoff vector 
so that the bargaining sets coincide with the core. However, any $x \in \mathbb{R}^{N}$ satisfying $x \leqslant 0$, but $x \nless 0$ (i.e., $x_{i}=0$ for some $\left.i \in N\right)$ belongs to $\mathcal{P} \mathcal{M}(N, V)$. However, $\mathcal{P} \mathcal{M B}(N, V)=\mathcal{C}(N, V)$.

For any finite nonempty set $N$ let $\Pi(N)$ denote the set of orderings of $N$, i.e.,

$$
\Pi(N)=\{\pi: N \rightarrow\{1, \ldots,|N|\} \mid \pi \text { is bijective }\} .
$$

Moreover, for $i \in N$ and $\pi \in \Pi(N)$, denote $P_{i}^{\pi}=\{j \in N \mid \pi(j)<\pi(i)\}$ and define, recursively, $x_{i}^{V, \pi} \in \mathbb{R} \cup\{-\infty\}, i=\pi^{-1}(1), \ldots, \pi^{-1}(|N|)$, by

$$
x_{i}^{V, \pi}= \begin{cases}0 & , \text { if there exists } j \in P_{i}^{\pi} \text { with } x_{j}^{V, \pi}=-\infty \\ \sup \left\{x_{i} \in \mathbb{R} \mid\left(x_{i}, x_{P_{i}^{\pi}}^{V, \pi}\right) \in V\left(P_{i}^{\pi} \cup\{i\}\right)\right\} & , \text { otherwise, }\end{cases}
$$

where $\sup \emptyset=-\infty$. The game is called marginal convex if, for all $\pi \in \Pi(N), x^{V, \pi} \in \mathcal{C}(N, V)$.

Example 4.4 Let $(N, V)$ be the 0-normalized game defined by Asscher (1976, Example 4.1), that is, $N=\{1, \ldots, 3\}$, for any $S \subseteq N$ with $|S|=2, x \in V(S)$ if and only if $x(S) \leqslant 210$ and, for all $k \in S, x_{k}+3 x_{\ell} \leqslant 450$, where $S \backslash\{k\}=\{\ell\}$, and $V(N)=\left\{x \in \mathbb{R}^{N} \mid x(N) \leqslant 300\right\}$. Then $x=(100,100,100) \notin \mathcal{C}(N, V)$, but, by a simple symmetry argument, $x \in \mathcal{M B}(N, V) \cap \mathcal{M}(N, V)$. Moreover, $\left\{x^{V, \pi} \mid \pi \in \Pi(N)\right\}=\{(0,150,150),(150,0,150),(150,150,0)\}=\mathcal{C}(N, V)$ so that $(N, V)$ is a non-leveled convex-valued marginal convex game.

A game $(N, V)$ is coalition merge convex if $(N, V)$ is superadditive and if the following conditions are satisfied:

(1) For any $\emptyset \neq S \varsubsetneqq T \subseteq N$ and any $x \in V(S)$ there exists $y \in V(T)$ such that $y_{S}=x$.

(2) For any $\emptyset \neq R, S, T \subseteq N$ with $S \varsubsetneqq T \subseteq N \backslash R$, any $x \in \partial V(S)$ satisfying $x_{i} \geqslant \max \{V(\{i\})\}$ for all $i \in S$, any $y \in V(T)$, and any $z \in \mathbb{R}^{R}$ such that $(x, z) \in V(S \cup R),(y, z) \in V(T \cup R)$.

According to Csóka, Herings, Kóczy, and Pintér (2011), a coalition merge convex game is marginal convex.

Remark 4.5 If $(N, V)$ is coalition merge convex and $x \in \mathbb{R}^{N}$, then $\left(N, V^{x}\right)$ is coalition merge convex. Hence our corollary may be extended: The unconstrained bargaining set and the MasColell prebargaining set of any coalition merge convex non-leveled NTU game coincide with its core.

Remark 4.6 The core of a cardinal convex game $(N, V)$ is nonempty provided that $V(N)$ is a convex set (Sharkey 1981). It can easily be verified that the excess game $\left(N, V^{x}\right)$ of a cardinal convex game $(N, V)$ is itself cardinal convex. However, even if $V(S)$ is convex for each $S \subseteq N$, then, as a union of convex sets, $V^{x}(N)$ may not be convex. We do not know if Corollary 4.2 holds for cardinal convex games with convex $V(N)$. 


\section{References}

Asscher, N. (1976): "An ordinal bargaining set for games without side payments", Mathematics of Operations Research, 1, $381-389$.

Aumann, R. J., And M. Maschler (1964): "The bargaining set for cooperative games", in Advances in Game Theory, ed. by M. Dresher, L. S. Shapley, and A. W. Tucker, vol. 52 of Annals of Mathematical Studies, pp. 443 - 476, Princeton, N.J. Princeton University Press.

Csóka, P., P. J.-J. Herings, L. Á. Kóczy, and M. Pintér (2011): "Convex and exact games with non-transferable utility", European Journal of Operational Research, 209, 57 - 62.

Davis, M., And M. MAschler (1967): "Existence of stable payoff configurations for cooperative games", in Essays in Mathematical Economics in Honor of Oskar Morgenstern, ed. by M. Shubik, pp. 39 - 52, Princeton, N.J. Princeton University Press.

GreenberG, J. (1985): "Cores of convex games without side payments", Mathematics of Operations Research, 10, 523 - 525.

Holzman, R. (2001): "The comparability of the classical and the Mas-Colell bargaining sets", International Journal of Game Theory, 29, 543 - 553.

Holzman, R., B. Peleg, and P. Sudhölter (2007): "Bargaining sets of majority voting games", Mathematics of Operations Research, 32, $857-872$.

Mas-Colell, A. (1989): "An equivalence theorem for a bargaining set", Journal of Mathematical Economics, 18, 129 - 139.

Maschler, M. (1976): "An advantage of the bargaining set over the core", Journal of Economic Theory, 13, $184-192$.

Maschler, M., B. Peleg, and L. S. Shapley (1972): "The kernel and bargaining set for convex games", International Journal of Game Theory, 1, 73 - 93.

Peleg, B. (1986): "A proof that the core of an ordinal convex game is a von NeumannMorgenstern solution", Mathematical Social Sciences, 11, 83 - 87.

Shapley, L. S. (1971): "Cores of convex games", International Journal of Game Theory, 1, 11 $-26$.

Sharkey, W. W. (1981): "Convex games without side payments", International Journal of Game Theory, 10, $101-106$.

Solymosi, T. (1999): "On the bargaining set, kernel and core of superadditive games", International Journal of Game Theory, 28, 229 - 240.

Vilkov, V. B. (1977): "Convex games without side payments", Vestnik Leningrad. Univ., 7, $21-24$, in Russian. 